\title{
Thoracoabdominal aortic aneurysm repair in Marfan syndrome: how we do it
}

\author{
Rana O. Afifi ${ }^{1,2}$, Akiko Tanaka ${ }^{1}$, Ibrahim Yazji ${ }^{1}$, Hazim J. Safi ${ }^{1,2}$, Anthony L. Estrera ${ }^{1,2}$ \\ ${ }^{1}$ Department of Cardiothoracic and Vascular Surgery, McGovern Medical School at the University of Texas Health Science Center at Houston \\ (UTHealth), Houston, TX, USA; ${ }^{2}$ Memorial Hermann Heart \& Vascular Institute, Houston, TX, USA \\ Correspondence to: Rana O. Afifi, MD. Department of Cardiothoracic and Vascular Surgery, McGovern Medical School at UTHealth, 6400 Fannin \\ St., Suite \#2850, Houston, TX 77030, USA. Email: Rana.o.afifi@uth.tmc.edu.
}

Submitted Jun 20, 2017. Accepted for publication Nov 06, 2017.

doi: 10.21037/acs.2017.11.04

View this article at: http://dx.doi.org/10.21037/acs.2017.11.04

\section{Clinical vignette}

A 17-year-old male patient was diagnosed at an outside hospital with an acute non-complicated type-B aortic dissection, 5 months prior to his recent admission. The patient underwent genetic workup that was positive for a PRKG1 gene mutation, which is associated with the genetic syndrome of familial thoracic aortic aneurysms and dissections (FTAAD). He was referred to our clinic due to an increase in aortic diameter. Computed tomography angiography (CTA) demonstrated a thoracoabdominal aortic aneurysm (TAAA), extending to the celiac access level, with largest diameter measuring $5 \mathrm{~cm}$. The patient was scheduled for an elective extent II TAAA repair. Although this specific patient does not have Marfan syndrome, the principle of our technique in relation to genetically associated thoracic aneurysms remains similar.

\section{Surgical technique}

\section{Preparation}

All patients undergo a CTA of the chest, abdomen and pelvis to define the extent of the TAAA (if renal function is impaired, then a non-contrast CT is performed). The patient is intubated with a double-lumen tube to block the left lung. Arterial line is placed in the right arm and intravenous catheters are placed in upper and lower limbs for blood transfusions. The patient is then placed in right lateral decubitus position, and a cerebrospinal fluid (CSF) drain is inserted between L3-L4 by the anesthesiologist.
Perioperatively, the CSF pressure is maintained at $10 \mathrm{mmHg}$. A team led by a neurologist/neurophysiologist performs neurophysiologic monitoring, including somatosensory evoked potentials (SSEPs) and motor evoked potentials (MEPs).

\section{Operation}

After induction of anesthesia and placement of CSF drain and electrodes for neurophysiologic monitoring, all patients are positioned in right lateral decubitus position, with the shoulder blades at the edge of the table at $90^{\circ}$ and the hip tilted at $60^{\circ}$ for access to both groins. The incision is made, which is tailored to the extent of the aneurysm. With regard to extent II, III, or IV, the skin incision begins just above the symphysis pubis and extends from the umbilicus to the costal cartilage. It is extended $2 \mathrm{~cm}$ below the angle of the scapula parallel to the vertebral border of the scapula. The latissimus dorsi and the serratus anterior muscles are cut. The pleural cavity is entered in the 6th intercostal space, the diaphragm is preserved, the retroperitoneal cavity is entered and abdominal organs are rotated medially, the renal temperature is monitored by placing a temperature probe in the cortex of the left kidney. The TAAA is now exposed after the Omni retractor (Integra LifeSciences, Plainsboro, New Jersey, USA) is placed. In young patients, and in patients with connective tissue disorders, we use the side-branched thoracoabdominal aortic graft (STAG) (Maquet Cardiovascular, Wayne, New Jersey, USA). An additional $14-\mathrm{mm}$ graft is attached to the main body to allow for reattachment or intercostal arteries (ICAs). The 
length of the graft is determined by stretching the graft with positioning of the celiac branch at the diaphragmatic hiatus. The left groin is opened and the common femoral artery is dissected. An 8-mm Dacron graft is sutured to the femoral artery in an end-to-side fashion.

We give the patient $1 \mathrm{mg} / \mathrm{kg}$ of heparin, we cannulate the inferior pulmonary vein is and the distal aortic perfusion is initiated. Distal aortic perfusion pressure is monitored through a line connected to the perfusion circuit (femoral cannula), and it is maintained between 60-80 mmHg. We cool the patient with mild "permissive" hypothermia (natural drifting down with bypass circulation), and maintain around $33^{\circ} \mathrm{C}$. If temperature drifts to lower temperature then we actively warm the patient to $35^{\circ} \mathrm{C}$ before coming off the bypass.

We use a sequential cross-clamping technique: the proximal clamp is placed distal to the left subclavian artery and the distal clamp is place in the mid descending aorta; we open the proximal descending aorta and divide the aorta circumferentially; we protect the esophagus with medial retraction with the suction tube and carefully dissect the posterior wall of the aorta away from the esophagus. The proximal end of the main body of the STAG is sutured to the aorta distal to the left subclavian artery in an endto-end fashion using 3-0 SH Prolene suture. After that is completed, the descending thoracic aorta is opened and patent ICAs T3-T7 are ligated with 2-0 silk figure-ofeight sutures. We move the distal clamp to the descending aorta at the level of the diaphragm, we occlude patent T8-T12 using \#3 Fogarty balloon catheters (Edwards Lifesciences, Irvine, California, USA). If there is no change in MEPS and SSEP, attention is addressed to the abdominal segment. The graft is passed through the hiatus of the preserved diaphragm. We further move the distal clamp to the infrarenal abdominal aorta, if the aneurysm extends further distally then we clamp the left iliac/common femoral artery. After identifying the visceral vessels, we perform renal perfusion using balloon tipped catheters with Houston solution (Ringer's lactate $1,000 \mathrm{~mL}, \mathrm{~K}+10 \mathrm{mEq}$, furosemide $5 \mathrm{mg}$, mannitol $8.5 \mathrm{~g}$ ) to maintain the left renal temperature below $20^{\circ} \mathrm{C}$. The initial bolus of Houston solution is usually around $500 \mathrm{~mL}$ and thereafter given intermittently to maintain the temperature. Tepid blood is infused to the SMA and celiac access. With no changes to MEPS, the distal anastomosis is completed, we place the patient in a head down position, flush the graft, de-air the graft by using an 18-gage needle, we then release the clamp, and raise the head of the patient slowly and establish pelvic circulation. Distal aortic perfusion continues to allow warming of the patient to $35^{\circ} \mathrm{C}$. The side branches are then cut to the appropriate length. A Carrel button patch is created for each branch and reconstructed individually to the side-arm branches of the graft with a 4-0 or 5-0 RB-1 Prolene running sutures. Before completion of each anastomosis, the perfusion catheters are removed and the grafts are de-aired. The 14-mm Dacron graft is prepared to re-anastomose the patent ICAs T8-T12. Even without loss of MEPS, we believe that reattachment of the patent ICAs T8-T12 helps reduce the risk of delayed paraplegia. The distal aortic perfusion is stopped and the cannulas are removed. Anticoagulation is then reversed and hemostasis is confirmed. Before closure, we now utilize cryoanalgesia by cryoablation of the intercostal nerves T4-T10.

\section{Completion}

We place 2-3 \#36 chest tubes. The pericostal space is approximated with two Vicryl sutures. In recent years, we place a sternal plate between the 6th and 7 th ribs for further stabilization of the chest wall. The muscular layers are approximated with \#1 PDS. The diaphragm and the linea alba are closed with \#1 Prolene suture. The skin is approximated with interrupted skin staples. Once sterile dressing is applied and the patient is hemodynamically stable, the patient is placed in the supine position and the double-lumen tube is exchanged for a single-lumen tube. The patient is then transferred to the intensive care unit for postoperative care.

\section{Comments}

Open TAAA repair in Marfan patients is the treatment of choice with good and acceptable outcomes $(1,2)$. Our management and surgical technique for TAAA has evolved over the past 25 years (3). We have demonstrated that the use of adjuncts for spinal cord protection decreases early paraplegia rates (4), and the decrease of postoperative acute renal failure by using cold visceral perfusion and nonischemic femoral arterial cannulation technique improves survival in patients with renal failure post TAAA repair $(5,6)$. These adjunct measures are utilized in all patients during open TAAA repair.

However, patients with connective tissue disorders require special considerations. The STAG is a pre-sewn, multiple-branched graft, which was designed by Dr. Hazim J. Safi in 1996 for use in repair of TAAA involving the 
visceral vessels. This technique allows for direct anastomosis to visceral vessels minimizing the amount of diseased aortic tissue left surrounding them. We demonstrated that despite the fact that the STAG technique requires more anastomoses compared to the inclusion technique, it can be performed with limited visceral ischemia as well as acceptable early outcomes (7). This is especially important in patients with connective tissue disease who are at a higher risk for developing patch aneurysms requiring redo surgery. A recent study demonstrated that $23.7 \%$ of our redo TAAA surgeries were performed in patients with connective tissue disorder (8). We use the reversed elephant trunk technique while performing the proximal anastomosis to facilitate any future proximal aortic repair.

In conclusion, we believe that open TAAA repair is the treatment of choice for patients with connective tissue disorder. We utilize adjuncts for spinal protection, including distal aortic perfusion, CSF drainage, moderate hypothermia, reattachment of ICAs T8-T12. While planning the repair, we pay special attention to minimizing the need for redo surgery as well as facilitating any future aortic repairs.

\section{Acknowledgements}

The authors acknowledge the contributions of Troy Brown for document editing and Chris Akers for illustrations.

\section{Footnote}

Conflict of interest: Dr. AL Estrera is a consultant for Gore and Maquet. The other authors have no conflicts of interest to declare.

Cite this article as: Afifi RO, Tanaka A, Yazji I, Safi HJ, Estrera AL. Thoracoabdominal aortic aneurysm repair in Marfan syndrome: how we do it. Ann Cardiothorac Surg 2017;6(6):709711. doi: 10.21037/acs.2017.11.04

\section{References}

1. Coselli JS, Green SY, Price MD, et al. Results of Open Surgical Repair in Patients With Marfan Syndrome and Distal Aortic Dissection. Ann Thorac Surg 2016;101:2193-201.

2. Mommertz G, Sigala F, Langer S, et al. Thoracoabdominal aortic aneurysm repair in patients with marfan syndrome. Eur J Vasc Endovasc Surg 2008;35:181-6.

3. Estrera AL, Sandhu HK, Charlton-Ouw KM, et al. A Quarter Century of Organ Protection in Open Thoracoabdominal Repair. Ann Surg 2015;262:660-8.

4. Safi HJ, Miller CC 3rd, Huynh TT, et al. Distal aortic perfusion and cerebrospinal fluid drainage for thoracoabdominal and descending thoracic aortic repair: ten years of organ protection. Ann Surg 2003;238:372-80; discussion 380-1.

5. Hassoun HT, Miller CC 3rd, Huynh TT, et al. Cold visceral perfusion improves early survival in patients with acute renal failure after thoracoabdominal aortic aneurysm repair. J Vasc Surg 2004;39:506-12.

6. Miller CC 3rd, Grimm JC, Estrera AL, et al. Postoperative renal function preservation with nonischemic femoral arterial cannulation for thoracoabdominal aortic repair. J Vasc Surg 2010;51:38-42.

7. De Rango P, Estrera AL, Miller C 3rd, et al. Operative outcomes using a side-branched thoracoabdominal aortic graft (STAG) for thoraco-abdominal aortic repair. Eur J Vasc Endovasc Surg 2011;41:41-7.

8. Afifi RO, Sandhu HK, Trott AE, et al. Redo Thoracoabdominal Aortic Aneurysm Repair: A SingleCenter Experience Over 25 Years. Ann Thorac Surg 2017;103:1421-8. 